Original

\title{
Dolor durante biopsia de próstata guiada por ultrasonografia transrectal: Comparación del uso o no de Xilocaína gel como anestésico local
}

\author{
Gilmer A. Díaz Pérez, Luis Meza Montoya, Carlos Morante Deza, Mariela Pow-Sang Godoy, \\ Víctor Destefano Urrutia
}

Instituto Nacional de Enfermedades Neoplásicas “Dr. Eduardo Cáceres Graziani” Lima, Perú

\begin{abstract}
Resumen
Introducción: La biopsia de próstata guiada por ecografía es el método de elección para el diagnóstico precoz del cáncer de próstata, es un método seguro cuya principal molestia es el disconfort que se siente durante el procedimiento.

Material y métodos: Desde julio del 2004 hasta marzo del 2005 se reclutaron 114 pacientes con indicación de biopsia de próstata bajo guía ecográfica los cuales se dividieron en dos grupos según la administración o no de xilocaína gel al $2 \%$ intrarrectal antes del procedimiento. Se comparó el dolor que sintieron durante la biopsia a través de una escala de dolor. Resultados: No hubo diferencia estadísticamente significativa entre los pacientes a quienes se administró anestésico local frente a quienes no se les administró. Por otro lado, el dolor fue mayor cuando se usó una aguja esterilizada que cuando se usó una aguja nueva.

Conclusiones: El uso de xilocaína gel al 2\% intrarrectal previa a la biopsia de próstata guiada por ecografía no disminuyó la sensación de dolor por el procedimiento en nuestros pacientes, el cual fue incrementado por el uso de agujas esterilizadas.
\end{abstract}

Palabras clave: Biopsia de próstata. Dolor.

Pain during transrectal ultrasound guided needle biopsy Of the prostate : comparison of the use or not of lidocaine Gel

\begin{abstract}
Introduction: The prostate biopsy guided by ultrasound is the method of choice for early diagnosis of prostate cancer, is a safe whose main trouble is that it feels discomfort during the procedure.

Material and methods: From July 2004 until March 2005 we prospectively studied 114 patients who underwent transrectal ultrasound guided needle biopsy of the prostate, they were divided in two groups: in one group we used lidocaína gel $2 \%$ inside the rectum before the procedure and in the other group we did not use it. We compared the pain that patients felt during the procedure with a pain scale.

Results: We did not find differences between both groups, but the patients in whom we used a new needle felt less pain than the patients in whom we used a re-esterilized one.

Conclusions: The use of lidocaine gel intrarectal $2 \%$ before a prostate biopsy guided by ultrasound did not diminish the sensation of pain from the procedure in our patients, which was increased by the use of sterilized needles.
\end{abstract}

Keywords: Prostate biopsy. Pain.

$\mathrm{L}$ a biopsia de próstata guiada por ecografía es el método de elección para el diagnóstico precoz del cáncer de próstata. Se considera un método seguro y usualmente se realiza en forma ambulatoria. Es un método invasivo que consiste en tomar muestras de ambos lóbulos prostáticos a través de un transductor sonográfico con una aguja de biopsia. La principal molestia que sienten los pacientes es el disconfort que representa (65-90\%) ${ }^{1-3}$ y el dolor por el procedimiento. Existen estudios en los que el bloqueo nervioso ha mostrado disminuir el disconfort de los pacientes ${ }^{4}$. En nuestro medio no se ha evaluado el dolor tras biopsia de próstata guiada por ecografía, y hemos observado que muchos de nuestros pacientes sienten dolor incluso previa administración de un anestésico local; por eso decidimos comparar mediante la escala de dolor cual es el dolor de nuestros pacientes en una biopsia de próstata guiada por ecografía aplicando anestesia local en un grupo de ellos y no aplicándola en el otro grupo. 


\section{MATERIAL Y MÉTODOS}

Desde el $1^{\circ}$ de julio de 2004 hasta el 31 de marzo de 2005 se reclutaron pacientes con indicación de biopsia prostática bajo guía ecográfica a quienes se les preparaba con dos enemas el día previo a la biopsia, administración de Amikacina 500 mg VIM el día anterior y el día de la biopsia, Ciprofloxacino $500 \mathrm{mg}$ VO cada 12 horas desde el día anterior a la biopsia hasta el día después de la biopsia. Todos debían tener un urocultivo negativo y en caso de estar tomando algún tratamiento anticoagulante se indicó su suspensión de acuerdo a los parámetros ya establecidos en la literatura. Se realizó la ecografía con un transductor de $6,5 \mathrm{MHz}$ y se utilizó una aguja número 18 nueva o esterilizada debido a los problemas de índole económica en nuestros pacientes que impiden usar una aguja nueva en cada caso, con la cual y través del transductor se tomaron doce muestras de la próstata (seis de cada lóbulo). Después de realizado el procedimiento se pidió al paciente indicar en una escala de dolor del 0 al 100 indicar en qué parte de la barra identificaba el dolor que había sentido por el procedimiento $(0$ en caso que no haya existido dolor hasta 100 que significa el máximo dolor imaginable). El número total de pacientes fue de 114 , y en forma alterna se les administró o no xilocaína gel al 2\% 5 minutos antes de realizar el procedimiento. En ningún caso se usó el gel ecográfico para facilitar el ingreso del transductor. En todos los casos se usó la xilocaína para este efecto. Se tomaron datos como la edad, el PSA total, la escala de dolor, el uso o no de anestésico, si la aguja fue o no esterilizada y si el resultado de la biopsia informó o no neoplasia maligna. Para el análisis estadístico se usó la media con la desviación estándar y para comparar ciertas características de ambos grupos se usó la prueba de t-student.

\section{RESULTADOS}

Un total de 114 pacientes constituyeron la muestra y de ellos a 67 se les administró anestesia y a 47 no se les administró. Las características del total de pacientes se presentan en las Tablas 1 y 2. Ambas poblaciones fueron comparables. Comparamos además ambas poblaciones en lo que respecta a la escala de dolor, considerando si la aguja fue nueva o esterilizada, el uso o no de anestesia y si la patología fue informada o no como neoplasia maligna. Encontramos que el uso de una aguja esterilizada ocasiona mayor dolor en
Tabla 1. Comparación de poblaciones.

\begin{tabular}{lccc}
\hline Caracteristica & Sin anestesia & Con antestesia & p \\
\hline $\begin{array}{l}\text { Edad } \\
\text { Media } \pm \text { DE }\end{array}$ & $67 \pm 9,32$ & $67,37 \pm 8,37$ & NS \\
$\begin{array}{l}\text { PSA } \\
\text { Media } \pm \text { DE }\end{array}$ & $11,55 \pm 7,37$ & $14,3 \pm 11,61$ & NS \\
$\begin{array}{l}\text { Aguja nueva } \\
\text { n/N(\%) }\end{array}$ & $7 / 47(14,9 \%)$ & $11 / 67(16,42 \%)$ & NS \\
$\begin{array}{l}\text { N. maligna } \\
\text { n/N(\%) }\end{array}$ & $12 / 47(25,53 \%)$ & $20 / 67(29,85 \%)$ & NS \\
\hline
\end{tabular}

Tabla 2: Características de los pacientes.

\begin{tabular}{lr}
\hline Edad & \\
Media +/- DE & $67,2 \pm 8,7$ años \\
PSA & \\
Media +/- DE & $13,2 \pm 10,1 \mathrm{ng} / \mathrm{m}$ \\
$\begin{array}{l}\text { Escala de dolor } \\
\text { Media +/- DE }\end{array}$ & $46,0 \pm 25,1$ punto \\
$\begin{array}{l}\text { Aguja reesterilizada } \\
\text { n (\%) }\end{array}$ & \\
$\begin{array}{l}\text { Anestesia } \\
\text { n (\%) }\end{array}$ & \\
$\begin{array}{l}\text { Neoplasia maligna } \\
\text { N (\%) }\end{array}$ & $67(58,2)$ \\
\hline
\end{tabular}

el paciente, hecho que fue también encontrado con el análisis de regresión, según vemos en las Tablas 3 y 4 .

\section{DISCUSIÓN}

En 1963 Takahashi y Ouchi fueron los primeros en usar la ecografía transrectal para evaluar la próstata $^{5}$, y en 1981 Holm y Gammelgaard describieron la biopsia de próstata transperineal guiada por ecografia ${ }^{6}$. Sin embargo, la comodidad del paciente fue baja debido al disconfort asociado con la biopsia en un área sensible. En 1989 Torp-Pedersen et al. reportaron los resultados de la biopsia prostática guiada por ecografía transrectal con una aguja número 18 con un ingenioso aditamento del transductor $^{7}$. Este método resultó de mejor comodidad para el paciente, debido a la relativa insensibilidad de la pared rectal y el rápido disparo de la fina aguja por un mecanismo automático.

En nuestras observaciones, una significativa proporción de los pacientes a quienes se les realiza una biopsia de próstata guiada por ecografía transrectal encuentra el procedimiento poco confortable y 
Tabla 3. Escala de dolor

\begin{tabular}{lcccc}
\hline & n & Media \pm DE & Test t & p \\
\hline $\begin{array}{l}\text { Aguja } \\
\text { reesterilizada }\end{array}$ & & & & \\
Si & 96 & $48,9+/-23,7$ & 3,025 & 0,003 \\
No & 18 & $30,1 \pm 26,9$ & & \\
& & & & \\
Anestesia & & & & \\
Si & 67 & $45,2 \pm 24,3$ & $-0,398$ & 0,692 \\
No & 47 & $47,1 \pm 26,3$ & & \\
& & & & \\
Neoplasia & & & & \\
maligna & & & & \\
Si & 32 & $46,4 \pm 20,2$ & 0,132 & 0,895 \\
No & 82 & $45,8 \pm 26,9$ & & \\
\hline
\end{tabular}

Tabla 4: Análisis de regresión

\begin{tabular}{lcccc}
\hline Factor & Coeficientes & $\begin{array}{c}\text { Error } \\
\text { estándar }\end{array}$ & Test t & Nivel p \\
\hline Constante & 46,197 & 18,863 & 2,449 & 0,016 \\
Edad & $-0,242$ & 0,266 & $-0,910$ & 0,365 \\
PSA & 0,037 & 0,229 & 0,161 & 0,872 \\
Aguja & 18,440 & 6,278 & 2,937 & 0,004 \\
\hline
\end{tabular}

el disconfort parece estar en relación al número de biopsias tomadas. En dos revisiones se encontró que entre el 65 al $90 \%$ de los pacientes reportaban disconfort $^{2-3}$. En los intentos por disminuir el disconfort del paciente se han utilizado técnicas de anestesia loco regional como el bloqueo nervioso prostático guiado por ecografía transrectal antes de la biopsia ${ }^{4}$, así como el uso de anestesia local con xilocaína en gel intrarrectal previa a la biopsia. El dolor asociado con la biopsia de próstata asienta predominantemente en la cápsula y el estroma prostático, donde hay una rica inervación de fibras autonómicas. Estos nervios autonómicos llevan sensibilidad visceral a la médula espinal. La inervación de la próstata se deriva de las raíces nerviosas de S2 a S5 y a través de la cadena simpática vía los plexos nerviosos presacro e hipogástrico. Estas fibras se ramifican en el plexo prostático y subsecuentemente viajan dentro de los pedículos vasculares de la próstata los cuales se localizan en el área posterolateral de la base prostática.

La biopsia de próstata con aguja guiada por ecografía es generalmente considerada como un procedimiento que no causa o causa un leve disconfort, y ha sido una práctica común realizarla sin anestesia o analgesia. Recientemente esta hipótesis ha siso cambiada por algunos. Cuando se evaluaron 81 pacientes por Inari $^{8}$ mostró que mientras la escala visual análoga del dolor referida por la mayoría de los pacientes fue de $3 / 10$, lo que sugiere que este procedimiento es sólo levemente inconfortable, el $16 \%$ de los pacientes tuvieron disconfort significativo (score mayor a 5/10). En suma 19\% de los pacientes reportaron que no desearía volverse a realizar una biopsia sin el uso de anestesia. En la serie de Clements $^{2}$ y Collins ${ }^{3}$, 30 y $22 \%$ de los pacientes respectivamente consideraron al procedimiento como significativamente doloroso. Parece ser que la edad, y no el número total de biopsias o el sitio específico de la biopsia determinan el disconfort individual en este procedimiento, por lo que los pacientes más jóvenes deberían ser advertidos que son más propensos a sentir más dolor con el procedimiento. Este subgrupo podría beneficiarse de algún analgésico a anestésico local. Existen reportes anecdóticos del uso de xilocaína gel o supositorios de xilocaína que podrían ser útiles en estos casos, mientras que Nash et al realizó el procedimiento previa anestesia con bloqueo nervioso regional de la próstata ${ }^{4}$. En el presente estudio casi el $67 \%$ de los pacientes reportaron haber presentado dolor mayor a 30/100 según la escala visual análoga, lo cual es una cifra mayor respecto a lo encontrado en la literatura. Es importante también tener en cuenta que la edad, en el análisis de regresión, no mostró ser un parámetro relacionado con una mayor sensación de dolor, sin embargo encontramos que el uso de una aguja esterilizada ocasionó más dolor que cuando se usó una aguja nueva, y en los pacientes en quienes se utilizó una aguja nueva tuvieron en la mayor parte de los casos una sensación de dolor menor a 30/100, lo cual indica un disconfort leve en comparación de aquellos en quienes se utilizó una aguja esterilizada donde la sensación de dolor fue moderada a severa. Es importante agregar que García Mediero et al. tampoco encontraron una diferencia significativa con el uso de xilocaína en gel, incluso administrado diez minutos antes del procedimiento, tiempo mayor al que nosotros esperábamos para realizarlo ${ }^{9}$. Nosotros no encontramos diferencias entre usar o no la xilocaína en gel intrarrectal previa a la biopsia, probablemente debido a que no bloqueamos con este método el paquete neurovascular que es el que lleva la sensibilidad y el cual se localiza en la cápsula y el estroma prostático. Debemos añadir aquí que la mayor sensación de 
dolor y disconfort en nuestros pacientes con respecto a los estudios de la literatura mundial se debería al uso de una gran cantidad de agujas esterilizadas, debido a el alto costo de las agujas nuevas para cada paciente, lo cual incrementaría el costo total de la biopsia haciéndola difícil de solventar para la mayoría de nuestros pacientes.

\section{CONCLUSIONES}

El uso de xilocaína gel al 2\% intrarrectal previa a la biopsia de próstata guiada por ecografía no disminuyó la sensación de dolor por el procedimiento en nuestros pacientes, el cual fue incrementado por el uso de agujas esterilizadas. Recomendamos que para hacer este procedimiento más confortable para nuestros pacientes debe usarse una aguja nueva en cada caso.

\section{Agradecimiento}

Al Sr. Alberto Minchón Medina quien se encargó del análisis estadístico.

\section{REFERENCIAS}

1. Rodríguez LV, Terris MK. Risks and complications of transrectal ultrasound guided prostate leedle biopsy: a prspective study and review of the literatura. J Urol. 1998;160(6 Pt1):2115-2120.

2. Clements R, Aideyan OU, Griffiths GJ, Peeling WB. Side effects and patient acceptability of transrectal biopsy of the prostate. Clin Rad. 1993;47(2):125-126.
3. Collins GN, Lloyd SN, Hehir M, McKelvie GB. Multiple transrectal ultrasound-guided prostatic biopsies-true morbidity and patient acceptance. Brit J Urol. 1993;71(4):460-463.

4. Nash PA, Bruce JE, Indudhara R, Shinohara K. Transrectal Ultrasound Guided Prostatic Nerve Blockade Eases Systematic Needle Biopsy of the Prostate. J Urol; 1996;155(2):607-609.

5. Takahashi, H. and Ouchi, T. The ultrasonic diagnosis in the field of urology (the first report). Proc Jap Soc Ultrasonics Med. 3: 7, 1963.

6. Holm HH, Gammelgaard J. Ultrasonically guided precise leedle placement in the prostate and the seminal vesicles. J Urol. 1981;125(3):385-387.

7. Torp-Pedersen S, Lee F, Littrup PJ, Siders DB, Kumasaka GH, Solomon MH, et al. Transrectal biopsy of the prostate guided with transrectal US: longitudinal and multiplanar scanning. Radiology. 1989;170(1 Pt 1):23-2.

8. Irani J, Fournier F, Bon D, Gremmo E, Doré B, Aubert J. Patien tolerance of transrectal ultrasound-guided biopsy of the prostate. Brit J Urol. 1997;79(4):608-610.

9. García Mediero JM, Martínez-Piñeiro Lorenzo L, Núñez Mora C, de Fata Chillón FR, Cruz Jimeno JL, Alonso y Gregorio S, de la Peña Barthel JJ. Uso de gel de lidocaína intrarectal en las biopsias transrectales ecodirigidas de próstata. Actas Urol Esp. 2003;27(10):793-796

Correspondencia autor: Dr. Gilmer A. Díaz Pérez

Urología Oncológica del Hospital General Santa Rosa.

Av. Bolívar s/n cuadra 8 - Pueblo Libre, Lima 21. Perú

Tel.: 511-6158200 - anexo 160

E-mail autor: gilmeruro@yahoo.com/gilmerqx@hotmail.com

Información artículo: Original - Cáncer de próstata

Trabajo recibido: octubre 2008

Trabajo aceptado: noviembre 2008 